FORMATION Formation emploi

Revue française de sciences sociales

136 | octobre-décembre 2016

Pêle-mêle

\title{
Une école « sociale active " pour des élèves acteurs, qualifiés et mieux orientés
}

An « active social » School for students active, qualified and well oriented "aktiven Sozial" Schule : für Schüler Spieler, qualifizierte und gut orientiert Los emprendedores de una Escuela "social activa" : volver a los alumnos actores, calificados y bien orientados

\section{Geneviève Mottet}

\section{(Q) OpenEdition}

Journals

Édition électronique

URL : http://journals.openedition.org/formationemploi/4902

DOI : 10.4000/formationemploi.4902

ISSN : 2107-0946

Éditeur

La Documentation française

Édition imprimée

Date de publication : 31 décembre 2016

Pagination : 7-28

ISSN : 0759-6340

Référence électronique

Geneviève Mottet, «Une école « sociale active » pour des élèves acteurs, qualifiés et mieux orientés », Formation emploi [En ligne], 136 | octobre-décembre 2016, mis en ligne le 31 décembre 2018, consulté le 30 octobre 2020. URL : http://journals.openedition.org/formationemploi/4902 ; DOI : https:// doi.org/10.4000/formationemploi.4902 


\section{Une école « sociale active » pour des élèves acteurs, qualifiés et mieux orientés}

GeneVIÈVE MotTet Docteure en sciences de l'éducation, université de Genève, FPSE (Faculté de psychologie et des sciences de l'éducation). Collaboratrice scientifique et chargée d'enseignement à l'université de Genève, SSED (Section des sciences de l'éducation)

Résumé

Une école « sociale active » pour des élèves acteurs, qualifiés et mieux orientés

Face au problème de l'employabilité des jeunes, l'objectif est ici de rendre compte des mesures entreprises, depuis le début des années 1990, par le ministère de l'Éducation à Genève. À partir d'une analyse des discours et dispositifs mis en place par trois ministres de l'Éducation successifs, l'article met au jour la genèse et l'essor d'une politique sociale active dans le domaine de l'éducation. Ainsi, la réforme s'est élaborée en deux temps, dont chacun comprend des référentiels d'action politique spécifiques : celui de l'" École active " $(1990$ - 2000) et celui de l'"École sociale active » (2000 - 2015).

Mots clés : politique de l'éducation, employabilité, système éducatif, orientation scolaireprofessionnelle, Suisse

Abstract

An «active social» School for students active, qualified and well oriented

This paper reports on measures taken by the Ministry of Education in Geneva since the early 1990s, regarding the problem of young people's employability. Based on an analysis of the political line and implemented policies of three successive Ministers of Education, the article reports on the genesis and development of an active social policy in the field of education. Thus, the reform was developed in two phases, each of which includes specific references to political action: that of the "Active School" (1990 - 2000) and that of the "Active Social School" (2000 - 2015).

Keywords: education policy, employability, education system, school guidance \& vocational guidance, Switzerland

Journal of Economic Literature: I 21, I 28

Traduction : Auteure. 
L'enjeu de cet article est de présenter comment, à Genève, les politiques éducatives se sont engagées, au cours des vingt-cinq dernières années, dans une réforme répondant à une logique d'employabilité et d'activation des jeunes en risque de désaffiliation scolaire et sociale. Cette réforme vise à lutter contre le décrochage scolaire des jeunes et à éviter qu'ils ne quittent prématurément l'école sans avoir terminé une formation de type secondaire II. Son objectif est de prévenir les risques futurs de chômage des jeunes, c'est-à-dire éviter le processus de désaffiliation sociale décrit par Castel (1995) qui correspond à la dissolution du lien social due à l'absence de travail et à l'isolement social. Comme le relève Blumer (1971), les problèmes sociaux suivent une carrière publique, des étapes que le sociologue peut documenter : l'émergence et la légitimation publique d'un problème, la mobilisation d'une série d'acteurs investis à le codifier et à définir son traitement, ainsi que la mise en ouvre de leurs recommandations dans des dispositifs déployés sur les terrains de l'intervention. Face au problème de la désaffiliation scolaire, notre objectif est ici de rendre compte du mécanisme des réformes du système éducatif à partir de mesures prises, depuis le début des années 1990, par trois ministres de l'Éducation genevois ${ }^{1}$. L'article met ainsi en lumière la carrière publique du problème de l'employabilité des jeunes depuis les années 1990 jusqu'à aujourd'hui, à l'aune de l'analyse des discours officiels de ces responsables politiques. Il porte essentiellement sur leur mobilisation dans la codification et la définition du traitement à appliquer, en mettant en exergue le processus d'émergence, puis de montée en puissance d'une nouvelle vision d'un problème (Muller, 2000). L'analyse propose de refléter le travail de mobilisation des ministres de l'Education (mobilisations qui peuvent suivre des stratégies différentes mais convergentes) et des entrepreneurs ${ }^{2}$, autour d'une réforme du système éducatif concernant l'employabilité des jeunes.

Plus précisément, nos observations (Mottet, 2013) nous amènent à considérer que, jusqu’à présent, la réforme s'est élaborée en deux temps, dont chacun comprend des référentiels d'action politique spécifiques : celui de l'« École active " (1990 - 2000), puis celui de l'«École sociale active » $(2000$ - 2015).

Le premier temps, à partir du début des années 1990, participe d'une volonté politique qui vise à augmenter le niveau de compétences des jeunes et leur potentiel d'employabilité en réalisant des transformations structurelles et pédagogiques, c'est-à-dire en favorisant l'élévation du niveau de formation et de compétences des jeunes ${ }^{3}$. La manière de codifier

1. En Suisse, les ministres de l'Éducation sont appelés chef(fe)s du Département de l'instruction publique (DIP).

2. Les débats sur l'école convoquent des arguments non seulement économiques et politiques, mais également éthiques et moraux. La construction de normes dans le domaine de la formation des jeunes relève du fruit du travail d' "entrepreneurs de morale " (Becker, 1985), c'est-à-dire d'acteurs qui se mobilisent pour faire adopter ou maintenir une norme sociale.

3. Selon Bonoli (2016), en Suisse, à partir de la deuxième moitié du XIXe siècle, la formation professionnelle poursuit deux finalités principales. D'une part, elle vise à assurer une main-d'œuvre qualifiée pour les entreprises et, d'autre part, elle œuvre pour une stabilisation socio-économique de la société. 
et de traiter le problème, durant cette période, s’incarnant dans une logique d'activation, nous avons nommé ce temps celui de l'"École active ».

Le second temps, à partir du début des années 2000, comprend une mobilisation politique qui confirme le projet d'activation de la période précédente, tout en intégrant explicitement une préoccupation concernant les problèmes sociaux et en s'engageant pour la cohésion sociale. Durant cette période, le ministère de l'Éducation investit la question sociale comme une donnée objective impactant le chômage et l'exclusion sociale. Il mobilise ce référentiel, délaissé depuis les années 1980 (Mottet et Bolzman, 2009 ; Boltanski et Chiapello, 1999), pour le problématiser à partir des populations à risque de désaffiliation scolaire précoce, notamment celle des élèves scolarisés dans des "territoires " défavorisés. Le ministère de l'Éducation s'investit particulièrement dans la qualification de ces publics en très grande difficulté en cherchant à améliorer leurs conditions d'accès à la formation professionnelle (Mottet, 2016). Il s'agit de déployer les moyens nécessaires pour qu'ils ne sortent pas du système scolaire sans qualification et qu'ils puissent être employables et actifs au sein de la société. Les discours et mobilisations du ministère de l'Éducation participent à l'élaboration du référentiel d'une "École sociale active " qui s’inspire des politiques sociales actives portées à l'échelle européenne dès la fin des années 1990.

Avant de rendre compte, empiriquement, des deux âges des politiques éducatives ici esquissées, précisons quelques éléments contextuels et méthodologiques dans l'encadré 1 .

\section{Encadré 1: Politiques éducatives et système de formation en Suisse (*)}

En Suisse, les ministres de l'Éducation sont des conseillers et conseillères d'État en charge du DIP (Département de l'instruction publique). L'organisation du système scolaire bénéficie historiquement d'une certaine autonomie vis-à-vis des autres cantons. Toutefois, les ministres de I'Éducation de chaque canton se réunissent dans le cadre de la CDIP (Conférence suisse des directeurs cantonaux de l'instruction publique), qui représente, en quelque sorte, le ministère national de l'Éducation. Il s'agit de développer des accords intercantonaux juridiquement contraignants et d'harmoniser les orientations des politiques éducatives entre les cantons.

Au degré secondaire II, la formation professionnelle est du ressort de la Confédération et des organisations du marché du travail. Il s'agit d'une formation duale entre l'école et l'entreprise, communément appelée " apprentissage ». Elle peut s'échelonner sur trois ou quatre ans et déboucher sur un CFC (Certificat fédéral de capacité) (1), ou sur deux ans et donner lieu à une AFP (Attestation fédérale de formation professionnelle) (2). La formation professionnelle représente la voie suivie par une forte proportion de jeunes (Wolter, 2010 ; Waardenburg, 2011).

Au début des années 1990, en raison de l'augmentation des exigences des employeurs en termes de niveau de formation attendu des apprentis, la CDIP s'est engagée à créer la maturité professionnelle (3) (Kiener \& Gonon, 1996), puis à institutionnaliser les Hautes écoles spécialisées (HES) en 1998, offrant la possibilité à l'« élite » des jeunes apprentis possédant ce diplôme de poursuivre une formation supérieure. 
En Suisse, en 2014, presque $15 \%$ des jeunes ont obtenu une maturité professionnelle (4). La maturité spécialisée est créée, en 2005, à Genève. Ce titre peut s'obtenir à l'issue d'une filière de formation en école de culture générale. La maturité gymnasiale (équivalent du baccalauréat généraliste) est la voie d'accès à l'université et aux écoles polytechniques fédérales (EPF). En Suisse, elle représente seulement environ $18 \%$ des titres de formation attribués pour le secondaire II (OFS, 2014, p. 36). À Genève, si le taux est plus élevé proportionnellement - $29 \%$ (SRED, 2014, p. 13) - il reste minoritaire par rapport aux différents diplômes relevant de la formation professionnelle. Le cas de Genève est particulièrement intéressant à analyser dans la mesure où ce canton, caractérisé par une forte attractivité de la voie gymnasiale (baccalauréat de fin d'études secondaires générales), a œuvré à la revalorisation de la voie professionnelle depuis le début des années 1990.

(1) : Équivalent du Certificat d'aptitude professionnelle (CAP) en France. Pour la correspondance entre diplômes suisses et français, voir : https://wirtschaftssprachen.hslu.ch/francais/2013/07/13/ equivalence-des-diplomes-suisses-et-francais/.

(2) : Équivalent du Brevet d'études professionnelles en France (BEP).

(3) : Équivalent du baccalauréat professionnel en France. Voir également le schéma de l'enseignement public à Genève en annexe électronique.

(4) : http://www.bfs.admin.ch/bfs/portal/fr/index/themen/15/06/dos/blank/05/02.html

${ }^{*}$ ) : L'encadré 1 et le schéma du système éducatif à Genève (en annexe électronique du présent article) visent à expliciter les particularités de la formation scolaire et professionnelle aux niveaux cantonal et fédéral.

Concernant les questions de contexte, on notera d'abord que les bornes temporelles de ces deux moments semblent découler de deux événements majeurs de l'intervention de l'Union européenne dans le domaine de l'éducation : le Traité de Maastricht (1992) et la Stratégie de Lisbonne (2000). Avec le premier, on assiste au lancement d'un « cadre commun " pour l'éducation, qui fonctionne comme un puissant et incontournable cadre de référence (Novoa, 2005). Rapprocher l'école et l'entreprise, lutter contre l'exclusion et former tout au long de la vie sont les concepts forts de ce cadre commun qui monte en puissance au milieu des années 1990. Les transformations qui seront engagées dans le second moment relèvent en partie de l'adoption, en 2000, d'un document stratégique du Conseil européen couvrant l'emploi, les réformes économiques et la cohésion sociale ${ }^{4}$. La Stratégie de Lisbonne est un événement clé qui marquera les politiques sociales et éducatives de ce début du siècle, en Suisse comme dans les autres pays européens. Selon Astier (2007), les objectifs d'un "État social actif», dont le concept émane au tournant des années 2000, sous l'impulsion du gouvernement belge, sont ceux qui visent à activer, reconnaittre, à se rapprocher, à personnaliser et à accompagner. Ils s'organisent autour d'un principe de gestion préventive et individualisée des risques sociaux et de participation citoyenne (Vielle, Pochet \& Cassiers, 2005). Dans différents champs d'intervention de

4. Précisons que même si la Suisse ne fait pas partie de l'Union européenne (UE), il existe un partenariat étroit officialisé par des traités bilatéraux et par la participation de responsables des politiques éducatives genevois et suisses aux instances européennes, telles que le Conseil de l'Europe. 
l'action publique, selon Orianne et al. (2008), on assiste au développement de " nouvelles " politiques publiques, organisées, pour l'essentiel, autour d'un triple mouvement d'individualisation, de conditionnalisation ${ }^{5}$ (Dufour $\&$ al. 2003) et de territorialisation des prestations de service public

\section{Encadré 2 : Méthode et matériaux de l'enquête}

D'un point de vue méthodologique, l'impact des politiques d'activation et d'employabilité sur les réformes engagées au sein des politiques éducatives sera analysé à l'aune des discours de trois ministres de l'Éducation genevois qui ont officié durant la période 1993 à 2015, à Genève. Il s'agit de M. Brunschwig Graf, entre 1993 et 2003 (Parti libéral) (1), de Ch. Beer, entre 2003 et 2013 (Parti socialiste), ainsi que d'A. Emery-Torracinta, en fonction depuis 2013 (Parti socialiste).

Cet article résulte de l'étude des discours des ministres de l'Éducation, ainsi que de l'analyse des contextes socio-historiques dans lesquels ils s'inscrivent. Les matériaux de l'enquête ont été recueillis grâce à une démarche de théorisation ancrée (2) (Glaser \& Strauss, 1967). L'analyse longitudinale des discours s'est faite à partir de différentes sources. Elle provient de l'étude des propos consignés dans les Mémoriaux du Grand conseil genevois (3), dans les recommandations du ministère (cantonal et national), dans les textes de loi, ainsi que dans des communiqués de presse et des rapports d'activité des responsables politiques. L'analyse rapporte également des propos de deux anciens ministres de l'Éducation recueillis dans le cadre de deux entretiens semi-directifs.

En outre, les discours des ministres sont mis en exergue ou cités par la presse. Nous avons donc procédé à une analyse médiatique des articles de presse d'un quotidien genevois portant sur les politiques éducatives durant la période évoquée. Environ 700 articles ont été collectés depuis 1990, informant sur ces acteurs, leurs positionnements, leurs discours et leurs pratiques, ainsi que sur le contexte dans lequel ils ont évolué.

L'article s'appuie enfin sur l'étude d'environ 250 sources documentaires provenant d'institutions et d'organisations multiples impliquées dans les politiques éducatives et la formation professionnelle : études du SRS (Service de la recherche sociologique), du SRED (Service de recherche en éducation), rapports de la CDIP (Conférence suisse des directeurs cantonaux de l'instruction publique), de l'OFFT (Office fédéral de la formation professionnelle et de la technologie), etc.

(1) : En 2009, le Parti libéral suisse (PLS) a fusionné avec le Parti radical-démocratique (PRD) formant le Parti libéral-radical (PLR) actuel dans lequel est engagée aujourd'hui cette personnalité politique. Le PLR est un parti de droite.

(2) : La théorisation ancrée peut se caractériser par la conceptualisation des données empiriques. La méthode est un aller-retour constant et progressif entre les données recueillies sur le terrain et un processus de théorisation.

(3) Les Mémoriaux du Grand Conseil contiennent la retranscription intégrale des sessions du Grand Conseil.

5. La conditionnalisation de l'action publique participe d'un système de droits et obligations réciproques entre l'individu et l'État. 
Les sources présentées dans l'article visent à révéler l'inspiration des réformes engagées. Elles mettent en lumière "le dialogue engagé entre textes et contextes" (Lassave, 1997). Les difficultés à rendre compte de cette articulation proviennent sans doute de la complexité à laquelle se confronte le chercheur lorsqu'il doit démontrer les liens directs et indirects entre les énoncés d'acteurs, les processus d'institutionnalisation et les transformations de contexte. Les données proviennent de sources diverses et couvrent une large période, ce qui peut interroger sur le choix de la démonstration portant sur un objet de large ampleur. Comme on le verra cependant, malgré ces limites, les sources s'articulent les unes aux autres et ont été choisies parce qu'elles explicitent, par la voix des acteurs étudiés, les raisons des institutionnalisations qui se sont opérées en direction des élèves à risque de désaffiliation scolaire et sociale. La portée de cette méthode et de ces matériaux est de permettre le repérage de référentiels d'action publique et de cerner les enjeux dans une interprétation globale du système.

Après cette introduction, nous présenterons l'analyse empirique des transformations des politiques de formation professionnelle à Genève, à partir des discours des ministres de l'Éducation qui se sont succédé au cours de ce quart de siècle. Nous verrons, dans un premier temps, comment le ministère de l'Éducation a promu une "École active " (19902000), puis, dans un second temps, une "École sociale active " (2000 à aujourd'hui). Les « mots d'ordre " des politiques éducatives de ces deux périodes et leurs enjeux seront présentés et introduits par des slogans des ministres de l'Éducation.

\section{L'«École active » (1990 - 2000)}

De 1990 à 2000, le rapport à la formation professionnelle se redéfinit de manière inédite. Au cours de ces années, les politiques éducatives vont promouvoir de nouveaux projets de formation pour les jeunes, l'objectif étant notamment de revaloriser la voie de l'apprentissage. Durant cette période, il s'agit d'améliorer le potentiel d'activation des élèves, de réorganiser les cursus de formation, ainsi que de repenser les formes de certification des différentes filières.

\subsection{L'apprentissage, le début d'un parcours}

Pour M. Brunschwig Graf, ministre genevoise de l'Éducation de 1993 à 2003, la création de la maturité professionnelle ${ }^{6}$, au milieu des années 1990, et celle des HES (Hautes écoles

6. Équivalent du Baccalauréat professionnel en France. 
spécialisées), à la fin de ces années, participent de la réforme la plus importante entreprise depuis la création du CO (Cycle d'orientation) ${ }^{7}$ dans les années 1960 :

"C'est la mise en place des hautes écoles spécialisées et de la maturité professionnelle, la vraie réforme de l'enseignement qui a eu des implications sur le plan cantonal, sur le plan fédéral et sur le plan intercantonal. Ça c'est clair. Elle touche aussi à l'école primaire. Elle touche à tout! [...] Si vous voulez, ça oblige à se dire: On n'est plus là comme dans les années 60 où on dit que faire un apprentissage, c'est la fin d'un parcours. Faire un apprentissage, c'est un début de parcours! Qui permet de faire une matu pro ${ }^{8}$ en même temps ou après, et qui permet d'avoir accès à une haute école spécialisée. " (Entretien, M. Brunschwig Graf, ministre genevoise de l'Éducation de 1993 à 2003, parti libéral)

Pour la ministre de l'Éducation, il s'agissait, au début des années 1990, d'amener de nouveaux débouchés à ceux qui, n'ayant obtenu qu'un CFC, se trouvaient limités dans leurs possibilités de formations futures. Dans le cadre de notre entretien, elle relève l'influence de la crise économique dans cette importante transformation du système éducatif, où les attentes des employeurs devenaient également plus fortes vis-à-vis du niveau de formation des jeunes. Dans ce contexte, les jeunes possédant une formation et un niveau scolaire faibles voyaient d'autant plus diminuer leurs possibilités de s'insérer dans la vie active. En conséquence, M. Brunschwig Graf dit s'être intéressée aux réformes à promouvoir afin que l'école permette aux jeunes des voies professionnelles de trouver leur place dans le monde du travail. Elle partage cette préoccupation avec les ministres de l'Éducation des autres cantons suisses qui s'accordent, dans le cadre de la CDIP, pour institutionnaliser de nouvelles filières visant à satisfaire les exigences intellectuelles et spécifiques de haut niveau (CDIP, 1995) :

"L'introduction des baccalauréats professionnels" et la promotion des Hautes écoles spécialisées proposent une alternative importante à la maturité gymnasiale et aux études universitaires. Afin d'être en mesure de remplir leur mandat, à savoir la formation de cadres hautement qualifiés, ces nouvelles filières devront satisfaire à des exigences intellectuelles et spécifiques de haut niveau. La CDIP recommande aux cantons de veiller notamment à la qualité de la formation qui conduit au baccalauréat professionnel. " (CDIP, 1995, p. 251)

La création de nouvelles structures dans la formation professionnelle supérieure voit paradoxalement se développer un discours politique engagé dans la limitation de l'accès aux études universitaires. Dans le cadre d'un entretien, la ministre de l'Éducation genevoise a tenu un discours critique sur le fait, qu’à cette époque, la maturité gymnasiale et l'univer-

7. Équivalent du secondaire I ; soit du collège au niveau de la classe d'âge, mais le cycle d'orientation fonctionne avec des filières.

8. Maturité professionnelle.

9. Il est intéressant de relever que la CDIP, soit l'équivalent du ministère suisse de l'Éducation, se référait, en 1995, au « baccalauréat professionnel » (diplôme français équivalent) au lieu de la «maturité professionnelle », intitulé officiellement choisi, en Suisse, depuis sa création en 1993. 
sité représentaient la voie de formation privilégiée pour les parents, les enseignants et les élèves. Le fait que le canton de Genève détenait le taux de maturité gymnasiale le plus élevé de Suisse constituait un problème pour elle, alors qu'il manquait une main-d'œuvre qualifiée. Elle déclare donc avoir dû œuvrer pour transformer les représentations publiques en développant d'autres champs de formation répondant aux exigences économiques propres à cette période. Son slogan - "Il n'y a pas de voie royale, mais des voies d'excellence $»^{10}$ éclaire la politique suivie de promotion de la formation professionnelle, qui passera également par la diminution du nombre d'universitaires ${ }^{11}$ et la réduction du temps accordé pour accéder à ces études et pour les achever.

\subsection{Des élèves davantage acteurs que consommateurs de leur formation}

Au cours de la transformation des systèmes de formation visant l'amélioration de l'employabilité des jeunes, on observe l'avènement des pédagogies actives au sein de l'école publique. De nouvelles attitudes vis-à-vis de l'apprentissage sont prônées par l'institution. Ainsi, la ministre de l'Éducation genevoise a soutenu la réforme de l'enseignement primaire au début des années 1990. Il s’agit pour elle de "produire " des élèves qui se comportent en acteurs et non en consommateurs de leur propre formation, et ce, dès les premières années d'école (Brunschwig Graf, 1999). Pour répondre à cette préoccupation, M. Brunschwig Graf s'est engagée, au début de son mandat, dans une rénovation de l'enseignement primaire en s'inspirant des principes de l'école active (Brunschwig Graf, 1994). Pour la ministre, les pédagogies actives promues dans ce contexte doivent contribuer à améliorer le système pour tous les élèves, qu'ils soient en difficulté ou non ${ }^{12}$. La pédagogie active a pour objectif de rendre l'élève acteur de ses apprentissages. Dans le cadre de notre entretien, elle souligne que le projet de rénovation vise une plus grande exigence vis-à-vis de chaque élève, puisqu'il s'agit d'en tirer le meilleur en y consacrant les moyens pédagogiques nécessaires, et ce, dès l'école enfantine (école maternelle) ${ }^{13}$.

Selon la politicienne, cette idée rejoint l'intention de changer le système pour que les élèves puissent investir les objectifs d'apprentissage le plus précocement possible, mais également pour détecter plus rapidement les élèves en difficulté et leurs offrir un suivi mieux adapté et individualisé. Le suivi sur plusieurs années de l'élève, notamment de l'élève se

\footnotetext{
10. Blog de M. Brunschwig Graf: Pourquoi je suis libérale. (http://www.brunschwiggraf.ch/text/35301.html) 11. Relevons qu'en Suisse, avec les EPF (Ecoles polytechniques fédérales), la certification universitaire est la plus élevée, à l'instar des Hautes écoles en France.

12. La rénovation porte sur trois axes au service des pédagogies actives : 1) individualiser les parcours de formation ; 2) développer le travail en équipes pédagogiques ; 3) placer les élèves au cœur de l'action pédagogique en s'inspirant des principes de l'école active.

13. Lors de son mandat, les deux premières années d'école enfantine n'étaient pas obligatoires. Les élèves pouvaient être scolarisés à partir de 4 ans, mais l'école n'était obligatoire qu’à partir de 6 ans. Aujourd'hui, la terminologie « école enfantine » n'existe plus. L'école primaire se déroule sur huit années..
} 
en difficulté, est considéré comme un projet central qui se déploiera autour de l'idée des cycles d'apprentissage et du travail en équipe pédagogique.

En présentant son projet de rénovation, la ministre relevait que tous les pays européens allaient dans le sens d'une telle réforme et que la rénovation s'inscrivait dans un courant général de modernisation des systèmes éducatifs (Brunschwig Graf, op. cit). La rénovation s'accompagne de dispositifs pédagogiques visant à développer, chez les élèves, des compétences d'auto-formation. Par exemple, une " pédagogie de contrat " dans laquelle l'élève fixe, avec son enseignant, des objectifs pour organiser son apprentissage et sa progression. Ainsi, l'élève est responsabilisé et amené à co-construire son projet de formation, notamment son orientation scolaire (Aebischer, 2012, pp. 60-61).

"Si l'on ne fait pas l'effort de voir quel bagage on peut donner pour que les bons réflexes viennent quand il s'agit d'apprendre ultérieurement, on produit des gens qui deviennent des handicapés de la formation continue, du perfectionnement professionnel et autre " (Entretien, M. Brunschwig Graf, ministre genevoise de l'Éducation de 1993 à 2003, parti libéral).

Schultheis (2001) fait valoir que "l'idée de life long learning s'impose comme modèle de quality management de soi et de bench marking individuel permanent " ${ }^{14}$ (p. 34). Plus précisément, il relève que la pensée néo-libérale d'employabilité semble offrir une solution à la question du chômage ; l'idée même de chômage devenant obsolète dès lors que le salarié se transforme en entrepreneur de sa propre force de travail. Cette nouvelle approche de la formation et de l'évaluation comporte ainsi de nouvelles exigences vis-à-vis des personnes. La promotion d'une "École active " par une ministre libérale de l'Éducation reflète son aspect paradoxal. Tout en étant généralement appréciées et promues par la gauche, les pédagogies actives se fondent parallèlement sur des principes inspirés du management du secteur privé. C'est bien en ce sens que M. Brunschwig Graf, qui a par ailleurs été directrice du Développement de l'économie suisse, a décidé d'institutionnaliser ces principes dans le cadre de la rénovation pédagogique. Aebischer (2012) relève, à propos de la loi d'orientation française de 1989, dont le texte ressemble fort aux principes de la rénovation du primaire genevois, que la tentation managériale se glisse largement dans les interstices pédagogiques et que, très souvent, les deux logiques (managériale et pédagogique) apparaissent intimement liées.

Durant ce premier âge, l' "École active ", les politiques éducatives développent des conditions structurelles et pédagogiques. Il s'agit de promouvoir les compétences qu'elles jugent nécessaires pour satisfaire les employeurs et ainsi lutter contre la hausse du chômage par la formation d'une main-d'œuvre qualifiée. Le référentiel de ce premier âge est celui de l'activation des publics par la transformation des systèmes de formation et des pédagogies.

14. L'idée maitresse d'employabilité véhicule un ensemble de maîtres-mots des sciences économiques tels que la formation tout au long de la vie, le fait de devenir entrepreneur de sa propre personne, d'agir plutôt que de réagir, et d'évaluation afin d'améliorer ses propres performances. Schultheis se réfère notamment aux travaux de Boltanski et Chiapello (op. cit.). 
La question sociale est quasiment absente des discours de la ministre de l'Éducation de cette période qui mobilise essentiellement le principe de l'individualité.

Avec le changement de ministre, en 2003, on observera le retour de la question sociale ou du référentiel socio-économique dans la construction de la réforme, élargissant le concept d'une "École active » à celui d'une "École sociale active ». On verra toutefois que le "retour des classes sociales " dans les propos du ministère de l'Éducation n'est pas uniquement dû à l'appartenance politique de Ch. Beer, ministre socialiste, mais qu'il s'imbrique dans le déploiement, à la fin des années 1990, d'un "État social actif " dans les pays européens.

\section{L'« École sociale active » (2000-2015)}

Selon nous, les réformes qui s'opèrent, à partir du début des années 2000, incarnent un nouvel " âge " des politiques éducatives d'activation. Cette nouvelle période est marquée par l'avènement d'un champ sémantique distinct. En effet, dans les discours, on assiste à la montée en puissance du référentiel de l'employabilité et de la cohésion sociale, correspondant au nouvel objectif de la Stratégie de Lisbonne. Ch. Beer, député socialiste qui succède, en 2003, à M. Brunschwig Graf, revendique l'amélioration des performances et de l'employabilité des élèves par un redressement de l'école, un meilleur encadrement et une meilleure orientation des élèves. Il intègre les notions d'équité et de cohésion sociale qui apparaissent dans le paysage politique dans la première moitié des années 2000 :

"Il est de ma responsabilité politique d'engager, dès aujourd'hui, le redressement de l'école publique genevoise afin qu'elle puisse jouer pleinement son rôle d'instruction publique et d'intégration, sans laisser aucun élève au bord de la route. " (Ch. Beer, ministre de l'Éducation, 2005, parti socialiste)

Voyons comment, dans le contexte des années 2000, les politiques éducatives genevoises développent leurs priorités et institutionnalisent des dispositifs en phase avec les politiques sociales actives portées à l'échelle européenne. Au début des années 2000, la notion de désaffiliation scolaire prend une place centrale dans les préoccupations des politiques éducatives. Le décrochage scolaire et le phénomène des non-qualifiés deviennent d'intérêt public. Il s'agit d'éviter que les élèves en grande difficulté ne se trouvent, par la suite, en situation de désaffiliation sociale, en tant que bénéficiaires de l'aide sociale. Les propositions du ministère de l'Éducation viseront à remédier à la question sociale des risques de désaffiliation scolaire par l'aide territorialisée et la sélection précoce, puis par le développement d'un suivi individualisé et des mesures d'accompagnement pour les jeunes en grande difficulté. 


\subsection{Renforcer l'aide territorialisée et développer l'école inclusive}

La conception de PISA $2000^{15}$ symbolise l'engagement des politiques éducatives ${ }^{16}$ dans une logique d'évaluation quantitative et qualitative des performances des jeunes qui permet la comparaison entre catégories de publics (selon l'origine, le milieu social, le sexe, l'âge, etc.). Le développement de ces indicateurs a conféré des moyens pour mieux cibler les populations à risque de désaffiliation scolaire et sociale. En Suisse, la question de la jonction entre le secondaire I et le secondaire II a été propulsée au centre des débats, suite aux résultats de cette enquête internationale, mais également aux conclusions de l'étude nationale TREE (TRansitions de l'École à l'Emploi) ${ }^{17}$ sur le parcours des élèves scolarisés, en Suisse, ayant participé à l'enquête PISA 2000. L'étude relevait que $10 \%$ des effectifs de cette classe d'âge étaient constitués de jeunes qui, quatre ans après leur sortie de l'école, ne suivaient pas de formation et n'avaient encore acquis aucun diplôme (Fibbi \& Mellone, 2010).

"Les recherches, les études et les enquêtes scientifiques dont nous disposons aujourd'hui autorisent des constats convergents sur les performances de l'école obligatoire : elles doivent être améliorées. Elles doivent l'être particulièrement pour les élèves les plus fragiles et les plus exposés" (Ch. Beer, 2005, ministre de l'Éducation, parti socialiste $)^{18}$.

De fait, en 2005, Ch. Beer, ministre de l'Éducation, présente son programme d'action dont l'objectif est de permettre la reconnaissance précoce des déficits des jeunes et d'y remédier dès l'école obligatoire. Il fixe ainsi " 13 priorités pour l'instruction publique genevoise ${ }^{19}$, qui s'inscrivent, selon ses dires, dans l'idée de cohérence et de qualité du système éducatif prônée afin de réduire les futurs risques de chômage des jeunes ${ }^{20}$.

En 2006, la création du REP (Réseau d'enseignement prioritaire) ${ }^{21}$, à Genève, constitue également une réponse du ministre de l'Éducation qui découle des constats de l'enquête PISA. L'innovation consiste à fournir des moyens financiers supplémentaires (encadrem-

15. Le but de PISA (Programme for International Student Assessment-Programme international pour le suivi des acquis des élèves) est de refléter la répartition des jeunes de 15 ans sur un continuum de compétences. Il s'agit de comparer les répartitions entre entités sociopolitiques et entre divers groupes et catégories de jeunes. L'enquête PISA vise également à comparer la qualité des systèmes éducatifs entre les pays.

16. M. Brunschwig Graf dit avoir eu un rôle fondateur dans le développement de PISA Suisse, en étant co-présidente du pilotage du système PISA (Entretien, M. Brunschwig Graf, cheffe du DIP entre 1993 et 2003, Parti libéral).

17. https://tree.unibas.ch/fr/home/

18. Propos de Ch. Beer, ministre de l'Éducation, publiés dans le quotidien genevois, Le Courrier, le 14 mai 2005.

19. http://edudoc.ch/record/619/?ln=en.

20. http://www.geneve.ch/dip/priorites/2/reinsertion_rupture_formation.asp.

21. La conception du REP semble, dès l'origine, se "calquer " sur les modèles contemporains des zones d'éducation prioritaires (ZEP) en France, soit tout à la fois le deuxième et le troisième âges des ZEP décrits par Rochex (2010). 
ments spécifiques) aux établissements scolaires primaires localisés dans des territoires présentant un pourcentage important d'élèves issus de milieux défavorisés. Réduire les risques d'une mauvaise intégration sociale et économique devient alors l'objet d'un partenariat entre l'État et les communes.

De même, la création du CATI-GE (Centre d'Analyse Territoriale des Inégalités), en 2009, témoigne de l'essor du thème de la cohésion sociale comme gouvernance publique. Elle résulte du souhait de Ch. Beer d'élaborer, avec le ministère des Finances (D. Hiler), une carte sociale du canton. La mise en réseau d'indicateurs statistiques issus de cette carte permettrait d'observer le développement des inégalités à Genève. L'innovation du CATI-GE vise à accentuer et coordonner l'effort public en lançant un plan d'investissement axé sur l'école, le logement, la culture, l'intégration et la sécurité :

"Nous voulons enfin une école qui tienne compte de la réalité des quartiers ou des communes socialement défavorisées en renforçant, là où il le faut, les moyens à disposition. " (Conseil d'État, 2009)

L'amélioration des compétences des élèves, notamment celles des jeunes à risque de désaffiliation scolaire, est alors une priorité du ministère national de l'Éducation (CDIP). Il met ainsi en place un accord intercantonal sur l'harmonisation de la scolarité obligatoire (CDIP, 2007) 22. L'encouragement précoce de l'enfant est également au cour de ce projet d'harmonisation qui rend obligatoire l'enseignement à quatre ans (au lieu de six) afin d'améliorer les chances initiales des enfants de réussir à l'école. L'harmonisation est pensée également entre le primaire et le secondaire I. Dans ce contexte, en 2011, Ch. Beer étend le REP au secondaire I.

En 2013, poursuivant le travail de son prédécesseur en matière de lutte contre le décrochage scolaire, la nouvelle ministre socialiste de l'Éducation, A. Emery-Torracinta (Parti socialiste), investit la question sociale à partir du projet d'école inclusive, thématique fortement promue dans les discours des politiques éducatives à partir des années $2010^{23}$ :

"L'école inclusive vise à offrir à chaque enfant et jeune la qualité d'encadrement et les moyens pédagogiques permettant son développement, quels que soient ses besoins, son handicap, son talent, son origine ainsi que ses conditions de vie économiques et sociales. » (Emery-Torracinta, 2015a, ministre de l'Éducation, Genève, depuis 2013)

La ministre promeut l'école inclusive afin de «maximiser le potentiel intellectuel, physique et social de chacun" et de lutter contre le décrochage scolaire (Emery-Torracinta, 2015a). Elle

22. Loi (10350) autorisant le Conseil d'État à adhérer à l'accord intercantonal sur l'harmonisation de la scolarité obligatoire (HarmoS) (C 1 06.0).

23. La thématique du projet relatif à «l'Organisation des prestations pour soutenir l'éducation inclusive " a été identifiée comme une priorité pour les pays membres de l'Agence européenne. Le projet a débuté en 2011 (Comité, 2014, "Présentation », La nouvelle revue de l'adaptation et de la scolarisation vol. 1, n 65, pp. 205206). 
précise que ce projet d'école inclusive est au service du principal défi de l'école genevoise consistant à augmenter le nombre de jeunes certifiés au sortir du secondaire II.

La politique éducative suivie par ces deux personnalités politiques, Ch. Beer et A. EmeryTorracinta, s'imbrique fortement dans une visée d'activation des publics à risque de désaffiliation scolaire et sociale.

\subsection{Qualifier tous les jeunes et améliorer l'orientation scolaire}

Parallèlement aux discours des politiques éducatives s'engageant dans le déploiement d'une "École sociale " par l'aide territorialisée et l'école inclusive, les politiques éducatives continuent à s'investir dans la promotion d'une "École active».

Cet investissement se traduit par une politique de qualification de tous les jeunes et par l'aide à l'orientation dans les formations adaptées aux différents publics. Le programme du ministre genevois se développe dans la lignée de la nouvelle loi fédérale sur la formation professionnelle (LFPr) de 2004. Dans ce cadre, l'attestation fédérale professionnelle (AFP) est institutionnalisée pour permettre aux élèves qui éprouvent des difficultés à suivre le cursus CFP, de s'intégrer néanmoins sur le marché du travail dans le cadre d'une formation initiale professionnelle de deux ans.

Le programme, présenté par le ministre de l'Éducation, Ch. Beer, s’inscrit également dans le cadre d'un projet national, élaboré en 2006, par l'ensemble des ministres cantonaux de l'Éducation en Suisse (CDIP). Son objectif est que $95 \%$ des jeunes puissent acquérir un diplôme du degré secondaire (CDIP, 2006 ; Confédération suisse, 2010). Le communiqué de presse de la CDIP stipule que les jeunes doivent perdre moins de temps à changer de voie ou à patienter dans un sas. Pour ce faire, l'objectif est d'organiser, à la fin de l'école obligatoire (secondaire I), des bilans de situation destinés à améliorer le choix d'une profession par les élèves. Le ministère national de l'Éducation (CDIP, 2006) demande à ce que les meilleures mesures (bonnes pratiques) en faveur des groupes à risque soient promues et précise que les mots-clés du projet doivent être le repérage des jeunes durant la scolarité obligatoire et la prise en charge individuelle. Ainsi, en décembre 2006, l'Office fédéral de la formation professionnelle et de la technologie (OFFT) développe le case management $2^{24}$ en "Formation professionnelle" (OFFT, 2007 ; Landert, 2011). La détection précoce des jeunes dont l'intégration professionnelle semble compromise bénéficie du soutien financier de la Confédération. Il s'agit d'assister les cantons dans la mise en place de projets susceptibles d'aider les jeunes à s'auto-responsabiliser et à achever une première formation post-obligatoire (OFFT, 2007). De fait, les case managers ont un certain

24. Le case management est une méthode d'accompagnement des jeunes en fonction de leurs besoins individuels et de leurs capacités. Il vise principalement à définir précisément et conjointement l'objectif du suivi, puis à accompagner la personne tout au long du processus d'intervention. 
nombre de tâches à effectuer, par exemple, contribuer à réduire au minimum le nombre de jeunes interrompant leur formation.

C'est dans le contexte des attentes et des institutionnalisations érigées au niveau national que Ch. Beer liste les initiatives prises par le ministère de l'Education :

"Depuis 2004, des projets et des prestations ont été développés au sein du DIP25. Nous nous limitons ici à les énumérer : bilan d'orientation en 8ème année ${ }^{26}$, coordination entre les différents services et partenaires concernés par l'accompagnement et le suivi des jeunes au niveau du secondaire I et II, "case management" genevois, Réussir + (suivi de l'apprentissage dual, OFPC ${ }^{27}$ ). " (Beer, 2007, p. 5)

Selon le ministre, la prise en charge doit débuter au cours de la scolarité obligatoire et se poursuivre au niveau de l'enseignement secondaire II. Il s'agit d'éviter le plus possible que les individus soient ultérieurement tributaires des assurances sociales (Beer, 2007). Depuis 2008, deux lois allant dans ce sens ont été adoptées à Genève : la première concerne la formation professionnelle et la seconde l'information et l'orientation scolaires et professionnelles ${ }^{28}$. Ces dispositifs visent à contrer les échecs des jeunes aux examens d'apprentissage et à remédier aux résiliations des contrats d'apprentissage par une orientation des jeunes en risque de désaffiliation scolaire et sociale. C'est en ce sens que Ch. Beer énonce ce slogan : "La fin des promus à rien", dans un communiqué de presse (Tribune de Genève, 16 juin 2009). Il s'agit d'améliorer l'insertion des jeunes en rupture de formation, notamment de les orienter dans une filière de transition "adéquate par rapport à leurs compétences, à leur projet et à leur situation scolaire " 29 (Beer, 2009).

"Dès la rentrée, trois filières seront disponibles. Les classes préparatoires aux écoles de commerce et de culture générale accueilleront les élèves "à pronostic scolaire favorable mais encore fragiles". L'année préparatoire intégrée aux Centres de formation professionnelle (voie normale vers les CFC) sera destinée à ceux forts d'un projet mais sans emploi. Et les "transitions professionnelles" s'adressent aux jeunes à la dérive, souffrant de problèmes de comportement et d'apprentissage. Un cadre "très directif" leur sera fourni " (Tribune de Genève, 16/06/2009, suite au communiqué de presse du ministre de l'Éducation, Ch. Beer, le $16 / 6 / 2009)$.

Ces mesures prises par le ministère genevois de l'Éducation, qui visent à promouvoir, dès l'école obligatoire, l'employabilité de chacun, s'accompagnent d'une volonté poli-

25. DIP : Département de l'instruction publique que nous appelons ici le ministère genevois de l'Éducation. 26. Avant la dernière année du secondaire I, CO, environ 15 ans, appelée aujourd'hui la 10ème année.

27. Office pour l'orientation, la formation professionnelle et continue (OFPC).

28. Lois (9917) et (9918).

29. Voir : (http://www.ge.ch/dip/priorites/2/adaptation_filieres_insertion.asp) (plus en ligne). 
tique de hiérarchiser plus fortement les filières du CO (Cycle d'Orientation, secondaire I) qui, à Genève, l'étaient déjà passablement.

En 2009, le CO a ainsi été l'objet d'initiatives et de votations populaires visant à le rendre plus sélectif. Un projet de loi pour un CO plus « exigeant et formateur pour tous " est accepté par 74,5\% des voix lors de la votation cantonale ${ }^{30}$. Il est ainsi décidé de diriger plus précocement les élèves dans des filières clairement hiérarchisées, tout en proposant des passerelles pour les élèves "désireux de progresser ". Pour les politiques qui se sont accordés sur le projet de loi, il s'agit d'éviter un "gâchis individuel et collectif, doublé d'un gaspillage de ressources publiques" (Barrillier, député radical au Grand Conseil genevois ${ }^{31}$, Tribune de Genève, 19 juin 2008). On entend ainsi prévenir les risques d'une mauvaise orientation dans des filières trop exigeantes en évaluant les potentiels liés aux compétences et en sélectionnant des débouchés en adéquation avec les profils considérés. La question du ciblage précoce des capacités des jeunes monte ainsi en puissance dans les intentions des politiques. Elle s'accompagne d'un encouragement, dès l'école obligatoire, à choisir une profession ou une formation. Le ministère national de l'Éducation indique ainsi que «les cantons sont invités à s'appuyer sur un dispositif cantonal visant à optimiser le choix d'une profession ou d'une école " (CDIP, 2011).

Ces recommandations se retrouvent dans la politique menée par l'actuelle ministre de l'Éducation, A. Emery-Torracinta (parti socialiste). Il s'agit de mieux orienter les élèves à la fin du $\mathrm{CO}$ pour éviter les réorientations en cascade (Emery-Torracinta, 2014). Son objectif prioritaire est : "Un jeune = une formation. " (Emery-Torracinta, ibid.). Ses slogans sont : "Viser la certification pour chaque jeune!", "Améliorer l'orientation scolaire ", "Valoriser la formation professionnelle " (Emery-Torracinta, 2015b). Aussi, suite à la mise en place, par son prédécesseur, d'une politique d'aide territorialisée, la nouvelle ministre répond aux injonctions de la CDIP par une politique promouvant l'orientation précoce des élèves, à partir d'un projet de formation "adapté à chaque élève ", dès l'enseignement primaire. L'actuel ministre genevois de l'Éducation (DIP) observe ainsi les effets statistiques de cette politique engageant les jeunes à se qualifier dans des filières professionnelles. A. Emery-Torracinta s'en félicite lors de sa Conférence de presse de la rentrée scolaire 2015 :

"Mieux orienter les élèves et valoriser la formation professionnelle. La volonté du département se traduit dans les faits. Les modifications réglementaires commencent à porter leurs fruits. Par rapport aux prévisions, il y a moins d'élèves inscrits à fin juin au Collège et à l'ECG ${ }^{32}$. Il y en a plus à l'École de commerce ${ }^{33}$. Le nombre total de places d'apprentis-

30. Pour plus de détails sur la question de l'hétérogénéité, versus de la sélection au cycle d'orientation, se référer notamment à Felouzis, Charmillot \& Fouquet-Chauprade (2013).

31. Organe législatif du canton.

32. Filières générales (voir en Annexe électronique le schéma de l'enseignement public à Genève).

33. Formation professionnelle (Voir en Annexe électronique le schéma de l'enseignement public à Genève). 
sage offertes en dual est en augmentation, ainsi que le nombre de jeunes ayant déjà signé un contrat. " (Emery-Torracinta, 2015a, ministre de l'Éducation depuis 2013, parti socialiste)

Le quotidien genevois, La Tribune de Genève, résume ainsi la conférence de presse : « $A$ Genève, plus d'apprentis, moins de collégiens ${ }^{34}$ "(Toninato, 2015).

\section{Conclusion}

Nous avons cherché à mieux comprendre les liens institutionnels qui se tissent, et se resserrent aujourd'hui, entre système éducatif et marché du travail. L'objectif a été de rendre compte du mécanisme des réformes du système éducatif au niveau local, à partir de mesures prises, depuis le début des années 1990, par trois ministres de l'Éducation genevois. L’article a empiriquement montré comment leurs projets politiques de réforme ont revisité l'ensemble du système éducatif en transformant les attentes de l'institution vis-à-vis des compétences à promouvoir, chez les élèves, dès le primaire. Nous avons présenté la construction d'une politique éducative d'activation de la formation et de l'employabilité comme étant le produit de deux périodes distinctes, aux référentiels d'action spécifiques.

Le premier, à partir du début des années 1990, participe d'une volonté politique de transformer structurellement et pédagogiquement l'école pour qu'elle réponde à une visée d'activation des élèves (de leur capacité à se former et à devenir plus performants). L'opération s'est effectuée grâce au repérage des meilleurs potentiels parmi les publics des voies professionnelles et par la promotion de cette élite dans des filières de formation supérieure. Cela a donné lieu à la création de la maturité professionnelle et des HES (Hautes écoles spécialisées).

Le second référentiel, à partir du début des années 2000, comprend une mobilisation politique en matière de gestion des risques de désaffiliation scolaire et sociale. Celle-ci confirme le projet d'activation de la période précédente, tout en intégrant explicitement le référentiel social. Il s'agit ainsi de diminuer les coûts engendrés par le versement de l'aide sociale, en prévenant les risques de désaffiliation (scolaire et sociale) des jeunes de quartiers ségrégués. En ce sens, les entrepreneurs d'une école "sociale active " promeuvent l'activation des publics à risque. Pour ce faire, ils traitent du problème à partir d'une logique territoriale de lutte contre les inégalités sociales, de cohésion sociale et d'inclusion, tout en orientant précocement ces publics vers des filières qualifiantes et professionnalisantes.

Suivant les observations d'Astier (2007), cet article révèle les ambiguïtés et injonctions contradictoires de ces politiques d'activation et de responsabilisation des publics

34. Le collège est la voie de formation qui mène à la maturité gymnasiale (baccalauréat général). 
à Genève, alors même que les politiques éducatives prônent une intervention sociale visant à reconnaître l'individu, à se rapprocher de lui, à lui fournir une aide personnalisée et à l'accompagner. Si l'État se définit aujourd'hui comme un État social et se veut actif dans ce sens, il promeut surtout un ensemble d'incitations à la mise au travail de catégories inactives. La question de la reproduction sociale (de classe, d'origine, de sexe) doit être questionnée dans le développement de ces transformations des politiques d'orientation professionnelle. Falcon (2016) montre ainsi que le système éducatif suisse, caractérisé par de nombreuses filières de formation professionnelle, joue un rôle important dans la persistance de ces inégalités. À la suite de Schultheis (2004), on assiste à une remise en cause de la politique d'accès aux études supérieures longues (université). De fait, les maturités gymnasiales et les maturités professionnelles ne sont pas destinées au même public et ne visent pas le développement des mêmes compétences. Les propos du conseiller fédéral en charge de l'économie, J. Schneider-Ammann, sur le lien entre compétitivité et types d'individus à promouvoir selon les besoins du marché, sont révélateurs d'une vision structurelle et hiérarchisante de la société promue :

«Et le conseiller fédéral de comparer la société à une pyramide. Le sommet, composé d'intellectuels, doit être soutenu par un large socle de personnes aux compétences avant tout manuelles. Il est important que ce principe soit intégré au système de formation. " (20 Minutes, 28 octobre $2012)^{35}$

Aussi, quels sont les rapports de force qui s'instaurent et qui impactent les orientations des jeunes vers des filières plus professionnalisantes, vers des maturités professionnelles ou spécialisées, plutôt que gymnasiales ? À la suite de Bonvin et Farvaque (2007), qui questionnent la place de la liberté réelle des demandeurs d'emploi à accéder à un emploi correspondant à leurs attentes, la question se pose aussi au niveau des élèves et de leur liberté réelle de s'orienter dans les différentes filières de formation proposées. Castel (2008) observe l'exercice d'une sorte de chantage au travail : "L'emploi est devenu un impératif catégorique quil faut accepter à n’importe quelles conditions : être un travailleur pauvre plutôt que d'être un mauvais pauvre qui ne travaille pas. " (p. 26)

Le cas de Genève, et plus largement de la Suisse, s'inscrit dans une perspective d'activation des publics qui participe d'un large consensus, porté à l'échelle européenne. Comme le souligne Novoa (2005), l'avènement et l'essor d'un Espace européen de formation constituent une "politique sans politique", dont l'action, conduite au niveau européen, est source d'inspiration au niveau national. Aussi, les mobilisations des ministres de l'Éducation genevois successifs, durant cette période, semblent suivre des logiques qui dépassent leur positionnement politique. D. Föllmi, qui a été ministre de l'Education à Genève, de 1985 à 1993, met lui-même en mots les ambivalences qu'il a perçues entre le type de

35. ATS (2012, 28 octobre), « Schneider-Ammann veut moins de maturités », 20 Minutes online (Quotidien romand). 
réforme des ministres de l'Éducation et leur positionnement politique. Évoquant la rénovation pédagogique promue par M. Brunschwig Graf (parti libéral), il relève ainsi :

"Parce que normalement, c'est quand même un projet que l'on aurait pu imaginer venant de milieux de gauche. Et Charles Beer, qui est socialiste, est en train de remettre "de l'autre dans l'autre sen's' ${ }^{36}$. C'est vraiment à l'envers. " (Entretien, D. Föllmi, chef du DIP entre 1985 et 1993, parti démocrate-chrétien)

Le poids des mots d'ordre européens n'est pas sans influencer le brouillage des positionnements politiques des ministres de l'Éducation. S'ils peuvent déployer leurs actions en développant leurs perspectives, ils le font dans le cadre de logiques globales (européennes, nationales, cantonales) qui les dirigent. M. Brunschwig Graf déclare ainsi :

"Chaque conseiller d'État [ministre de l'Éducation], quand il arrive à la direction du Département [ministère cantonal de l'Éducation], a une situation à gérer qui part du point $X$ et qui va au point $Y$, et puis entre deux, il prend les mesures nécessaires. Alors des fois, il décide quill changera tout, mais souvent il s'appuie sur ce qui a été fait et il développe et il prend d'autres initiatives, etc. [...]. C'est-à-dire, un besoin, dans le vocabulaire, de paraître original et différent, mais dans la réalité, le système, il est beaucoup plus têtu et il se construit dans la durée. " (Entretien, M. Brunschwig Graf, ministre de l'Éducation entre 1993 et 2003, parti libéral)

Il s'agit bien d'interroger la complexité du système, ses mutations et ses logiques d'action, tout en rendant compte de la complexité des relations entre "l'acteur et le système " (Friedberg \& Crozier, 1977). À la suite de Muller (2000), nous pouvons dire que si les politiques éducatives régionales ou nationales, et les acteurs qui les produisent, suivent leur propre chemin pour réformer leur système scolaire, cela s'opère dans un nouveau paysage commun, structuré par un modèle éducatif global véhiculé par des instances européennes et internationales (Conseil de l'Europe, Unesco, etc.). De fait, face à une "nébuleuse réformatrice ${ }^{37}$ (Topalov, 1999), il s'avère particulièrement complexe - en montrant empiriquement la genèse et les transformations des réformes - de statuer sur l'ordre réel des liens de causalité dans les mots d'ordre des politiques éducatives. Et ce d'autant que certains acteurs genevois (ministres de l'Éducation et chercheurs en éducation au sein du ministère) sont (ou ont été) engagés dans des groupes de travail nationaux et supranationaux. Il serait intéressant d'analyser plus finement la dynamique qui s'opère dans le va-et-vient de ces acteurs entre les diverses instances. Il serait également

36. Le ministre de l'Éducation, D. Föllmi, veut dire ici que le ministre Ch. Beer, socialiste, en créant des directions d'établissement dans l'enseignement primaire, a suivi une politique en lien avec des propositions généralement faites par la droite.

37. Les mots d'ordre sur l'employabilité des jeunes sont construits par des acteurs en réseaux (et des réseaux d'acteurs) souvent non concertés et engagés progressivement dans un travail de problématisation et de régulation. 
pertinent d'analyser les effets des réformes engagées par les politiques éducatives sur les acteurs de terrain que sont les enseignants et les élèves.

\section{Bibliographie}

Aebischer S. (2012), « Réinventer l'école, réinventer l'administration. Une loi pédagogique et managériale au prisme de ses producteurs ", Politix, 2(98), pp. 57-83.

Astier I. (2007), Les nouvelles règles du social, Paris, PUF.

Barrillier G. (2008), "Les politiques veulent un Cycle d'orientation exigeant ", Tribune de Genève, p. 12.

Becker H. (1985), Outsiders. Études de sociologie de la déviance (trad. de l'américain par J.-P. Briand et J.-M. Chapoulie), Paris, A.-M. Métailié.

Beer Ch. (2013), Rapport d'activité Charles Beer, Conseiller d'État, Congrès du Parti socialiste genevois, 9 mars, Genève.

Beer Ch. (2009), La formation professionnelle : une réponse à la crise, Rentrée scolaire 2009, Genève, Conférence de presse du Département de l'instruction publique.

Beer Ch. (2007), Aide à l'insertion des jeunes gens en rupture de formation. Action publique concertée, Rapport du Conseil d'État, août, Genève, DIP.

Beer Ch. (2005), « Un plan d'action pour réunifier l'école et combattre les inégalités », Le Courrier, Genève, 14 mai.

Blumer H. (1971), «Social Problems as Collective Behavior», Social Problems, (17)3, pp. 298-306.

Boltanski L., Chiapello È. (1999), Le nouvel esprit du capitalisme, Paris, Gallimard.

Bonoli L. (2016) «Aux origines de la fonction sociale de la formation professionnelle suisse. Une logique de reproduction sociale ", Formation Emploi, 133, pp. 17-34.

Bonvin J.-M., Farvaque N. (2007), «L'accès à l'emploi au prisme des capabilités, enjeux théoriques et méthodologiques ", Formation Emploi, 98, pp. 9-22.

Brunschwig Graf M. (1999), "Chercheurs et décideurs : une relation d'interdépendance ”, in Lurin J. \& Nidegger Ch. (textes rassemblés par), Expertise et décisions dans les politiques de l'enseignement, Actes du colloque de Penthes, février 1998, Genève, SRED, pp. 19-23.

Brunschwig Graf M. (1994), Individualiser les parcours de formation, apprendre à mieux travailler ensemble, placer les enfants au cour de l'action pédagogique. Trois axes de rénovation de l'école primaire genevoise, Genève, Département de l'instruction publique. 
Castel R., Enriquez E., Stevens H. (2008), « D’où vient la psychologisation des rapports sociaux? ", Sociologies pratiques, 17, pp. 15-27.

Castel R. (1995), Les métamorphoses de la question sociale, Paris, Fayard.

CDIP (2011), Recommandations. Transition scolarité obligatoire - degré secondaire II, 28 octobre, Berne, Conférence suisse de cantonaux de l'instruction publique.

CDIP (2007), Accord intercantonal sur l'harmonisation de la scolarité obligatoire (concordat HarmoS), 14 juin, Berne, Conférence suisse de cantonaux de l'instruction publique.

CDIP (2006), Davantage de diplômés du secondaire II, Communiqué de presse, Berne, CDIP, 13 novembre.

CDIP (1995), Recommandations et décisions, Berne, CDIP.

Confédération suisse (2010), Nouvelle loi fédérale sur la formation professionnelle. Bilan après six ans d'application, rapport du Conseil fédéral, Berne, Conseil fédéral.

Conseil d'État (2009), Discours de Saint-Pierre, prestation de serment du Conseil d'État, 7 décembre.

Dufour P., Boismenu G. \& Noël A. (2003), L'aide au conditionnel : la contrepartie dans les mesures envers les personnes sans emploi en Europe et en Amérique du Nord, Bruxelles, PIE-Peter Lang.

Économie Suisse (2008), « HarmoS : harmonisation de l'école obligatoire », Dossier politique, $\mathrm{n}^{\circ} 17$.

Emery-Torracinta A. (2015a), Le DIP sur le chemin de l'école inclusive, Conférence de presse du 18 août 2015, Genève, DIP.

Emery-Torracinta A. (2015b), Rapport d'activité-Année 2014, Congrès du parti socialiste genevois, 14 mars.

Emery-Torracinta A. (2014), Donnons des moyens à l'école, Congrès du Parti socialiste genevois, 22 mars, Genève.

Falcon J. (2016), «Les limites du culte de la formation professionnelle : comment le système éducatif suisse reproduit les inégalités sociales " Formation Emploi, 133, pp. 33-53.

Felouzis G., Charmillot S., Fouquet-Chauprade B. (2013), "Comment organiser l'enseignement secondaire obligatoire ? Une étude de cas à Genève ", Revue Suisse de Sociologie, Special Issue Change and Reforms in Educational Systems and organisazion, 39(2).

Fibbi R., Mellone V. (2010), Jeunes en transition de l'école obligatoire au degré secondaire II : quelle participation pour les parents ?, Étude réalisée sur le mandat de la CDIP (Conférence suisse des directeurs cantonaux de l'instruction publique), Neuchâtel, Université et SFM. 
Friedberg E., Crozier M. (1977), L'acteur et le système, Paris, Seuil.

Glaser B. \& Strauss A. (1967), The Discovery of Grounded Theory. Strategies for Qualitative Research, Chicago, Aldine de Gruyter.

Kiener U. \& Gonon Ph. (1996), La maturité professionnelle comme illustration de la politique suisse en matière de formation professionnelle, Berne, Projet National de Recherche (PNR) 33 sur l'efficacité de nos systèmes de formation, Rapport de valorisation.

Landert Ch. (2011), Projet national Case management « Formation professionnelle, Rapport sur l'évaluation de la mise en œuvre, Département fédéral de l'économie (DFE), Berne, Office fédéral de la formation professionnelle et de la technologie (OFFT).

Lassave P. (1997), Les sociologues et la recherche urbaine dans la France contemporaine, Toulouse, Presses universitaires du Mirail.

Mottet G. (2016), «Agir contre la précarité des jeunes en rupture de formation : l'émergence d'une nouvelle catégorie d'action des politiques éducatives en Suisse ", Sciences et actions sociales [en ligne], 3.

Mottet G. (2013), À l’ "Ecole de la diversité», Enquête sur la fabrique d'une politique éducative, thèse de doctorat en sciences de l'éducation, université de Genève.

Mottet G. \& Bolzman C. (2009), L'École et l'élève d'origine étrangère : genèse d'une catégorie d'action publique, Genève, IES éditions.

Muller P. (2000), «L'analyse cognitive des politiques publiques : vers une sociologie politique de l'action publique ", Revue française de science politique, 2, pp. 189-208.

Novoa A. (2005), "Les états de la politique dans l'espace européen de l'éducation », in Lawn M., Novoa A. (coord.), L'Europe réinventée. Regards critiques sur l'espace européen de l'éducation, Paris, L'Harmattan.

OFFT (2007), Le case management "formation professionnelle ». Principes et mise en auvre dans les cantons (ou comment donner une chance à tous les jeunes), Berne, OFFT, Office fédéral de la formation professionnelle et de la technologie.

OFS (2014), Mémento statistique de la Suisse 2014, Neuchâtel, OFS, Office fédéral de la statistique.

Orianne J.-F., Draelants H. \& Donnay J.-Y. (2008), « Les politiques de l'autocontrainte », Education et sociétés, 2(22), pp. 127-143.

Rochex J.-Y. (2010), "Les trois “âges” des politiques d'éducation prioritaire : une convergence européenne ?", in Ben Ayed C. (dir.), L'école démocratique. Vers un renoncement politique?, Paris, Armand Colin. 
Schultheis F. (2004), «La stratégie européenne de l'emploi : entre lutte contre la précarité des jeunes et production d'un habitus flexible ", Swiss Journal of Sociology, 30(3), pp. 303-318.

Schultheis F. (2001), "Splendeurs et misères de la jeunesse sous le règne du nouvel esprit du capitalisme ", in SRED, Jeunesse d'aujourd'hui. Analyse sociologique de la jeunesse et des jeunes dans une société en mutation rapide, Genève, Éditions du SRED.

SRED (2014), Memento statistique de l'éducation à Genève. Édition 2014, Genève, Service de la recherche en éducation.

Toninato A. (2015), "À Genève, plus d'apprentis, moins de collégiens », Tribune de Genève, Genève, 18 août.

Topalov C. (Dir.) (1999), Laboratoires du nouveau siècle : la nébuleuse réformatrice et ses réseaux en France (1880-1914), Paris, EHESS.

Vielle P., Pochet P. \& Cassiers I. (2005), L'État social actif : vers un changement de paradigme?, Bruxelles, PIE-Peter Lang.

Waardenburg G. (2011), "Le système suisse de diplômes ", in Millet M., Moreau G. (Dir.), La société des diplômes, Paris, La Dispute.

Wolter S. (Dir.) (2010), L'éducation en Suisse, Rapport 2010, Aarau, SKBF/CSRE. 